\title{
Spatial Asymmetries Undermine Also the Short Forms of the Judgement of Line Orientation Test
}

\author{
Barbara Treccani and Roberto Cubelli \\ University of Trento
}

\begin{abstract}
Objective: The Judgment of Line Orientation (JLO) test is one of the most used tasks for the assessment of visuospatial perception. However, JLO items show a left-right structural asymmetry that interacts with the ipsilesional attentional biases of brain-damaged patients, that is, the main target population for which the test is intended, and undermines the test's validity. Left hemisphere-damaged patients are favored by the way the stimulus lines are distributed in the original items, whereas right hemisphere-damaged patients are favored by the opposite distribution, obtained by mirror reversing the items (Treccani, Torri, \& Cubelli, 2005). Here we aimed to analyze the short forms of JLO available in the literature, which are often presented as preferable alternatives to the full form. Method: Characteristics of the items of these short forms were scrutinized. By reanalyzing data from Treccani et al. (2005), we also investigated the impact of these characteristics on brain-damaged patients' performance. Results: Seven of the 8 analyzed short forms proved to be even more asymmetric than the full form (e.g., they have a different number of left and right lines), whereas the remaining one, which uses a flexible item-selection criterion, leads to unpredictable results. Like in the full form, these asymmetries affect brain-damaged patients' performance. Conclusions: The presence of spatial asymmetries in JLO items cannot be neglected anymore in the development of any JLO form, given their impact on performance and the resulting detrimental effect on the accuracy and validity of the measurement. We propose to select 5-10 items among those of the original set and present them together with their mirror images to obtain a left-right balanced JLO short version.
\end{abstract}

\section{General Scientific Summary}

This study shows that, in all the published short forms of the most popular neuropsychological test for assessing the ability to judge line orientations, the different possible line slopes and the segment types are not equally distributed in the left and right halves of the test items. This disfavors right brain-damaged patients, who tend to privilege the ipsilesional side when processing the test stimuli.

Keywords: judgment of line orientation, visuospatial perception, neuropsychological assessment , unilateral brain damage, unilateral neglect

The Judgment of Line Orientation (JLO) test (Benton, Hamsher, Varney, \& Spreen, 1983) is one of the most widely used measures for the assessment of visuospatial perception (Qualls, Bliwise, \& Stringer, 2000; Lezak, Howieson, Bigler, \& Tranel, 2012; van der Berg \& Ruis, 2017). It examines the ability to discriminate angular relationships between stimulus line segments by requiring participants to identify the orientation of pairs of angled lines on a multiple-choice display comprising 11 numbered lines (see Figure 1, Panel A).

JLO is used in many different clinical and research settings and is often described as especially successful in detecting visuospatial

This article was published Online First December 27, 2018.

Barbara Treccani and Roberto Cubelli, Dipartimento di Psicologia e Scienze Cognitive, University of Trento.

Correspondence concerning this article should be addressed to Barbara Treccani, Dipartimento di Psicologia e Scienze Cognitive, University of Trento, Corso Bettini, 31, 38068 Rovereto, Italy. E-mail: barbara.treccani@ unitn.it deficits that follow cerebral lesions in the right hemisphere. Indeed, right hemisphere-damaged (RHD) patients usually have been found to show poorer performances on JLO than left hemisphere-damaged (LHD) patients (Benton, Varney, \& Hamsher, 1978; Hamsher, Capruso, \& Benton, 1992; Tranel, Vianna, Manzel, Damasio, \& Grabowski, 2009).

However, Treccani et al. (2005) showed that factors unrelated to the ability of judging spatial orientations can affect brain-damaged patients' performance on JLO: the presence of contralesional neglect, which is more frequent and severe following right than left hemisphere lesions (Cubelli, 2017), and the left-right structural asymmetry of JLO stimulus displays, which advantages LHD patients. Treccani et al. (2005) showed that patients with left neglect are significantly more impaired on this test than patients without neglect (either with left or right hemisphere lesions). They also pointed out that there are relevant differences in the perceptual features of JLO left- and right-sided lines. In the left and right halves of JLO stimulus displays, there is a different number of both horizontal lines (lines 1 and 11) and lines with a slope close to 


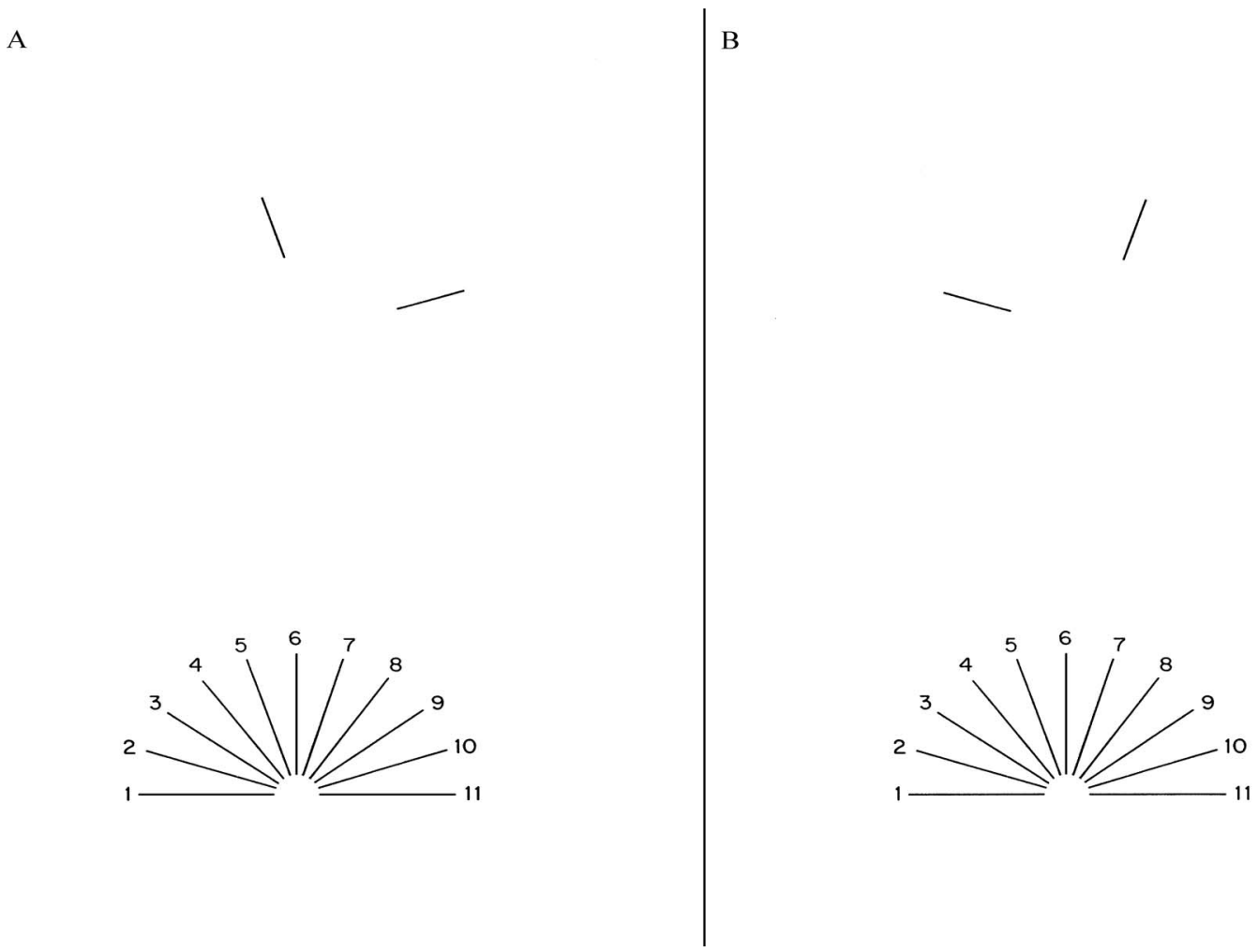

Figure 1. Item 1 from Form V (Panel A) of the Judgment of Line Orientation test and its mirror-reversed version (Panel B).

either the horizontal or vertical, and a different proportion of the three stimulus segments (proximal, middle, or distal) representing the response choice lines. As shown by the results obtained by Treccani et al. (2005) in their Experiments 1 and 2, these perceptual asymmetries make the lines on the right side more difficult to judge than those on the left side and disadvantage RHD patients, who tend to privilege the right (ipsilesional) side.

In Experiment 1, Treccani et al. (2005) found that normal participants were more accurate in judging left- than right-sided lines of JLO items. This was due to the perceptual features of JLO left-sided lines (i.e., their slopes and segment type) and not to their position (i.e., the fact that they are on the left side). Indeed, these lines were judged more accurately even when they were presented on the right. When participants were presented with the mirrorreversed versions of JLO items (i.e., items obtained by rotating the original JLO items around the vertical axis; see Figure 1, Panel B), they were more accurate with right-sided lines. When original and mirror-reversed items were considered together, a left-right balanced JLO version was obtained (with the same lines on the left and right), and no difference between performances in the two spatial sides was observed.

In Experiment 2, Treccani et al. (2005) administered the original and mirror-reversed versions of JLO items to patients with unilateral brain lesions. Results showed that both LHD and RHD patients were more accurate with ipsilesional lines than with the contralesional ones, even when only patients without clinical signs of contralesional neglect were taken into account. The conjoint effect of spatial biases due to unilateral cerebral lesions (i.e., the preference for ipsilesional stimuli, which is frequently observed even when contralesional stimuli are not actually neglected; Colombo, De Renzi, \& Faglioni, 1976) and the structural asymmetry of JLO results in opposite behaviors of LHD and RHD patients without neglect on the two item versions. Performance was more accurate for LHD patients than RHD patients with original items and for RHD patients than LHD patients with mirror-reversed items.

In a subsequent study, Treccani and Cubelli (2011) compared JLO performance of RHD and LHD patients without neglect with that of neurologically intact participants matched for gender, age, and years of education. With the original items, RHD patients showed more impaired performances than their controls, whereas no difference was observed between LHD patients and controls. With the mirror-reversed items, the opposite pattern emerged: LHD patients' performances were more impaired than those of their controls, whereas there was no difference between RHD patients and controls. Thus, RHD and LHD patients appeared to show normal or defective performance, depending on the JLO version they underwent. Notably, when both original and mirrorreversed items were considered together, no significant differences between LHD and RHD patients without neglect, and between patients and their controls, were observed. These results suggest that the selective impairment of RHD patients in line orientation judgment is an artifact. Clearly the perceptual features of some lines of JLO items are such as to favor either LHD or RHD patients when presented, in the original and mirror-reversed versions, re- 
spectively, on the side toward which patients devote more attention.

Treccani et al. (2005) and Treccani et al. (2011) concluded that to assess appropriately the ability to judge line orientations, an extended, left-right balanced version of JLO, comprising the original items intermixed with their mirror-reversed images, should be used. In this extended version, the same lines would be presented on the left and on the right side; thus, patients with unilateral attentional biases would face all the JLO lines in both their favored and disfavored sides.

However, in both clinical and research settings, there are often time constraints that make this 60 -item test version unsuitable for routine use (Moore et al., 2015; Spencer et al., 2013). Actually, even the standard version of the test (with the 30 original items) has long administration times (up to $15 \mathrm{~min}$ ), which can be frustrating, particularly for older examinees. Because of these reasons, several JLO short forms have been proposed (Calamia, Markon, Denburg, \& Tranel, 2011; Mount, Hogg, \& Johnstone, 2002; Qualls et al., 2000; Venderploeg, LaLone, Greblo, \& Schinka, 1997; Winegarden, Yates, Moses, Benton, \& Faustman, 1998; Woodard et al., 1998).

Spencer et al. (2013) compared JLO short forms by analyzing their internal consistency and concluded that the short form by Calamia et al. (2011) is the most suitable form for either clinical or screening purposes. According to Spencer et al. (2013), most JLO short forms are adequate only for screening purposes (i.e., they are brief but involve great measurement errors), whereas that proposed by Calamia et al. (2011) demonstrates good reliability and could be included in neuropsychological batteries as a stand-alone measure.

However, the analysis done by Spencer et al. (2013) does not take into account the spatial features of the lines presented in the left and right halves of the stimulus displays. In most short forms, an arbitrary criterion of item selection is used (e.g., only the first 10 items or only the odd items). In Calamia et al.'s (2011) study, a flexible criterion is used. They adopted an item response theory (IRT) approach: Items are reordered according to difficulty estimates, participants start from the 16th most difficult item, and basal and ceiling rules of six items passed and failed, respectively, are applied. It follows that a variable number of items is administered to each participant.

Neither a fixed, arbitrary criterion nor a flexible criterion based on the number of consecutive correct/incorrect responses guarantees the same level of difficulty in the two spatial sides of the presented items. In fact, a selection based on these criteria leaves open the possibility of even more serious left-right asymmetries: Not only might the selected items be asymmetric in terms of difficulty, but they also might even have a different number of left and right lines.

The aim of this study was to analyze the features of left and right lines of the items of the eight JLO short forms published in the literature. In particular, we aimed to establish whether these short forms are asymmetric, just like the full JLO forms, and to analyze the impact of the possible asymmetries on the performances of unilateral brain-damaged patients. To this end, we reanalyzed the data of Treccani et al. (2005) and compared performances on the original and mirror-reversed versions of the items selected for the different short forms.

In Treccani et al.'s (2005) study, all patients with visuospatial neglect showed a severe JLO impairment. Their accuracy for the lines in the affected hemispace was very low and they did not benefit from the different distributions of stimulus lines in the mirror-reversed version of JLO. Indeed, they showed defective performances on both the (original and mirror-reversed) versions of the test. Accordingly, for the present study, only patients who did not show any clinical signs of neglect on the neuropsychological evaluation were selected from Treccani et al.'s sample.

In our previous study (Treccani et al., 2005), the mere (subclinical) leftward or rightward spatial biases induced by unilateral lesions (i.e., spatial biases that do not result in an overt manifestation of neglect; Colombo et al., 1976), coupled with the asymmetrical distribution of JLO items, were shown to modulate LHD and RHD patients' performances on the JLO full form. In the present study, we aimed to investigate whether similar modulations of JLO performance can be observed with the short forms of the test.

\section{Method}

\section{Participants}

Treccani et al. (2005, Experiment 2) examined 37 right-handed patients who had suffered a stroke and with a lesion confined to either the left or right hemisphere. Neither the size nor the intrahemispheric site of the lesion was taken into account. The cerebrovascular lesion was documented by computed tomography or magnetic resonance imaging scan and supported by clinical neurological evaluation. From these 37 patients, we selected those without clinical signs of neglect (see Treccani et al., 2005 for details): 20 LHD and 10 RHD patients. Demographic and clinical characteristics are reported in Table 1. The two groups did not differ in age and distance from the onset of lesion (for both $t$ test comparisons, $p \geq .7$ ). The composition of the two groups in terms of gender was not significantly different either (Yates-corrected $\left.\chi^{2}=0.02, p=.89\right)$. Yet RHD patients had an educational level significantly higher than that of LHD $[t(28)=2.10, p<.05]: 11.9$ and 8.4, respectively. Accordingly, when RHD and LHD performances were compared, the number of years of education was used as a covariate.

In Treccani et al. (2005), patients' cognitive level was evaluated by means of two standardized tests and equivalent scores (i.e., five ordinal categories, ranging $0-4$, in which age-education adjusted scores were classified) were used to compare the LHD and RHD groups. Given the possible verbal impairment in LHD patients, equivalent scores from a nonverbal intelligent test (Colored Progressive Matrices; Basso, Capitani, \& Laiacona, 1987; Raven, 1965) were used as the index of global cognitive level for these patients. In contrast, equivalent scores from a verbal test (Verbal

Table 1

Demographic and Clinical Characteristics of Participants: Mean (and Range)

\begin{tabular}{cccc}
\hline Groups & Age & Years of education & Days from onset \\
\hline LHD $(n=20)$ & $59.65(36-72)$ & $8.40(5-18)$ & $480.40(25-1953)$ \\
RHD $(n=10)$ & $61.20(39-75)$ & $11.90(5-18)$ & $563.70(18-3016)$ \\
\hline
\end{tabular}

Note. LHD $=$ left hemisphere-damaged patients; RHD $=$ right hemisphere-damaged patients. 
Judgments Test; Spinnler \& Tognoni, 1987) were used for the RHD group. A Mann-Whitney $U$ test revealed that the RHD and LHD patients selected in the present study did not significantly differ in general intelligence $(\mathrm{z}=0.92 ; p=.35)$ : Their mean intelligence equivalence scores were 2.6 and 2.2 , respectively.

\section{Materials and Procedure}

Treccani et al. (2005) tested patients with a modified version of the JLO consisting of five practice items and 60 test items: The 30 original JLO items, intermingled with their mirror-reversed images. Only the items included in the eight JLO short forms examined by Spencer et al. (2013; see Table 1) and their corresponding mirror-reversed images were considered for the analyses in the present study. From the original full Form V (Benton et al., 1983), the short forms included the following items: odd or even items (Venderploeg et al., 1997; Mount et al., 2002); items 1-10, 1-20, 11-30 (Winegarden et al., 1998); items 2, 6, 7, 9, 12, 16, 17, 19, 20, 21, 22, 24, 26, 28, 30 (Form Q, Qualls et al., 2000); items 1, 3, 4, 5, 8, 10, 11, 13, 14, 15, 18, 23, 25, 27, 29 (Form S, Qualls et al., 2000). The items of the IRT-based short form devised by Calamia et al. (2011) were identified following the method described by these authors.

\section{Results}

In Table 2, the JLO full form and the eight short forms considered by Spencer et al. (2013) are listed. Characteristics of the items and of the lines composing the items are shown. In Calamia et al.'s (2011) IRT-based short form, the exact items administered to a given examinee depend on which items are correctly identified by him/her. Accordingly, for this JLO form, average values based on the items that would have been administered to our sample of brain-damaged patients are shown. Following Calamia et al.'s method, the mean number of items that would have been presented is 21.2: 20.45 items to LHD patients and 22.7 items to RHD patients, $t(28)=1.123, p=.27$.
Overall, the eight short forms appear to be largely asymmetric. Just as in the full form, the proportion of proximal, middle, and distal segments, the number of segments coupled with a segment of the same type, and the number of lines with angles intermediate between the major axes (i.e., the lines that are the most difficult to judge; Appelle, 1972; Treccani et al., 2005) are different in the left and right halves of the items.

In addition, at variance with the full form, in all short forms the total number of lines, the lines coupled with a vertical line (i.e., a segment particularly easy to judge, as observed by Treccani et al., 2005), and those coupled with a line lying on the same side (i.e., the monolateral items) differ between the two hemispaces.

Actually the differences between the two hemispaces does not seem substantial for Calamia et al.'s (2011) short form. However, with this form opposite asymmetries were observed for different patients (more lines on the left for some patients and more lines on the right for other patients), which balanced each other when considering mean values. For 19 of the 30 examined patients, either the left or the right lines of the administered items outnumber the lines on the other side by at least two units (and up to nine). For 22 patients, the difference between left and right lines concerns at least one difficult-to-judge line.

To investigate the possible impact of these asymmetries on performance, the JLO score was calculated for both the original and mirror-reversed versions of all short forms by using the JLO standard scoring method (Benton et al., 1983). Response to an item was scored as correct if both stimulus lines were accurately identified. Percentages of correctly answered items were used to compare the eight short forms because of their different number of items. These percentages were analyzed by means of analysis of variance with group (LHD, RHD) as between-subjects factor and two within-subjects factors: JLO form (full, odd, even, 1-10, 1-20, 11-30, Q, S, IRT-based forms) and item version (original and mirror-reversed). To control for the possible effect of the number of years of education (which differs between LHD and RHD

Table 2

Characteristics of the Full Form of the Judgment of Line Orientation (JLO) Test and Its Short Forms

\begin{tabular}{|c|c|c|c|c|c|c|c|c|c|c|c|c|c|c|c|c|c|}
\hline \multirow[b]{2}{*}{ Source } & \multirow[b]{2}{*}{ JLO form } & \multicolumn{3}{|c|}{ Lines (total) } & \multicolumn{2}{|c|}{$\begin{array}{l}\text { Between major } \\
\text { axes lines }\end{array}$} & \multicolumn{2}{|c|}{$\begin{array}{l}\text { Distal (coupled } \\
\text { with distal) }\end{array}$} & \multicolumn{2}{|c|}{$\begin{array}{l}\text { Type of segment } \\
\text { Medial (coupled } \\
\text { with medial) }\end{array}$} & \multicolumn{2}{|c|}{$\begin{array}{l}\text { Proximal (coupled } \\
\text { with proximal) }\end{array}$} & \multicolumn{2}{|c|}{$\begin{array}{c}\text { Monolateral } \\
\text { items }\end{array}$} & \multirow{2}{*}{$\begin{array}{l}\text { Bilateral } \\
\text { items }\end{array}$} & \multicolumn{2}{|c|}{$\begin{array}{l}\text { Item with a } \\
\text { vertical line }\end{array}$} \\
\hline & & Left & Right & Central & Left & Right & Left & Right & Left & Right & Left & Right & Left & Right & & Left & Right \\
\hline Benton et al., 1983 & Full & 28 & 28 & 4 & 9 & 10 & $12(9)$ & $10(8)$ & $8(7)$ & $9(7)$ & $8(6)$ & $9(8)$ & 7 & 7 & 12 & 2 & 2 \\
\hline \multirow[t]{2}{*}{$\begin{array}{l}\text { Venderploeg et al., } \\
1997\end{array}$} & Odd & 16 & 12 & 2 & 7 & 4 & $7(6)$ & $5(4)$ & $5(4)$ & $4(4)$ & $4(3)$ & $3(2)$ & 5 & 2 & 6 & 0 & 2 \\
\hline & Even & 12 & 16 & 2 & 2 & 6 & $5(3)$ & $5(4)$ & $3(3)$ & $5(3)$ & $4(3)$ & $6(6)$ & 2 & 5 & 6 & 2 & 0 \\
\hline \multirow{3}{*}{$\begin{array}{l}\text { Winegarden et al., } \\
1998\end{array}$} & $1-10$ & 12 & 6 & 2 & 2 & 0 & $5(5)$ & $4(3)$ & $4(4)$ & $2(2)$ & $3(3)$ & $0(0)$ & 3 & 0 & 5 & 1 & 1 \\
\hline & $1-20$ & 15 & 22 & 3 & 3 & 5 & $9(8)$ & $5(3)$ & $7(7)$ & $8(7)$ & $6(5)$ & $2(2)$ & 6 & 3 & 8 & 2 & 1 \\
\hline & $11-30$ & 16 & 22 & 2 & 7 & 10 & $7(4)$ & $6(5)$ & $4(3)$ & $7(5)$ & $5(3)$ & $9(8)$ & 4 & 7 & 7 & 1 & 1 \\
\hline \multirow{2}{*}{ Qualls et al., 2000} & Q & 13 & 14 & 3 & 4 & 6 & $6(5)$ & $2(2)$ & $4(4)$ & $5(4)$ & $3(3)$ & 7 (7) & 3 & 4 & 5 & 2 & 1 \\
\hline & $\mathrm{s}$ & 15 & 14 & 1 & 5 & 4 & $6(4)$ & $8(6)$ & $4(3)$ & $4(3)$ & $5(3)$ & $2(1)$ & 4 & 3 & 7 & 0 & 1 \\
\hline Calamia et al., 2011 & IRT based & 20.1 & 20.0 & 2.3 & 7.3 & 7.6 & $8.7(6.2)$ & $6.9(5.7)$ & $5.9(5.1)$ & $5.6(4.0)$ & $5.5(3.8)$ & $7.5(6.7)$ & 5.3 & 5.1 & 8.5 & 1.0 & 1.3 \\
\hline
\end{tabular}

Note. For each form, the total number of lines, the number of lines with angles intermediate between the major axes and the number of proximal, middle, and distal segments in either hemispace (left/right) are reported. Numbers of monolateral items (i.e., both lines in the same hemispace), bilateral items (i.e., one line in the left hemispace and the other in the right hemispace), and items composed of a central, vertical line (number 6) coupled with either a left or right line are also shown. For Calamia et al.'s, 2011short form, average values are shown (see text for details). 
Table 3

Number (and Percentages) of Left (LHD) and Right (RHD) Hemisphere-Damaged Patients Classified as Either Unimpaired or Impaired According to Their Age-and Gender-Corrected Score (CutOff >20) on the Full Form of the Judgement of Line Orientation (JLO) Test and on Six JLO Short Forms (See Text for Details)

\begin{tabular}{|c|c|c|c|c|c|c|}
\hline \multirow[b]{3}{*}{ Source } & \multirow[b]{3}{*}{ JLO Form } & \multirow[b]{3}{*}{ Version } & \multicolumn{4}{|c|}{$N$ patients } \\
\hline & & & \multicolumn{2}{|c|}{ LHD } & \multicolumn{2}{|c|}{ RHD } \\
\hline & & & Unimpaired & Impaired & Unimpaired & Impaired \\
\hline \multirow[t]{2}{*}{ Benton et al., 1983} & \multirow[t]{2}{*}{ Full } & Original & $12(60 \%)$ & $8(40 \%)$ & $3(30 \%)$ & $7(70 \%)$ \\
\hline & & Mirror reversed & $13(65 \%)$ & $7(35 \%)$ & $6(60 \%)$ & $4(40 \%)$ \\
\hline \multirow[t]{4}{*}{ Venderploeg et al., 1997} & \multirow[t]{2}{*}{ Odd } & Original & $13(65 \%)$ & $7(35 \%)$ & $4(40 \%)$ & $6(60 \%)$ \\
\hline & & Mirror reversed & $12(60 \%)$ & $8(40 \%)$ & $6(60 \%)$ & $4(40 \%)$ \\
\hline & \multirow[t]{2}{*}{ Even } & Original & $13(65 \%)$ & $7(35 \%)$ & $4(40 \%)$ & $6(60 \%)$ \\
\hline & & Mirror reversed & $14(70 \%)$ & $6(30 \%)$ & $7(70 \%)$ & $3(30 \%)$ \\
\hline \multirow[t]{2}{*}{ Winegarden et al., 1998} & \multirow[t]{2}{*}{$11-30$} & Original & $12(60 \%)$ & $8(40 \%)$ & $4(40 \%)$ & $6(60 \%)$ \\
\hline & & Mirror reversed & $11(55 \%)$ & $9(45 \%)$ & $6(60 \%)$ & $4(40 \%)$ \\
\hline \multirow[t]{4}{*}{ Qualls et al., 2000} & \multirow[t]{2}{*}{ Q } & Original & $11(55 \%)$ & $9(45 \%)$ & $5(50 \%)$ & $5(50 \%)$ \\
\hline & & Mirror reversed & $13(65 \%)$ & $7(35 \%)$ & $6(60 \%)$ & $4(40 \%)$ \\
\hline & \multirow[t]{2}{*}{$\mathrm{S}$} & Original & $13(65 \%)$ & $7(35 \%)$ & $3(30 \%)$ & $7(70 \%)$ \\
\hline & & Mirror reversed & $13(65 \%)$ & $7(35 \%)$ & $6(60 \%)$ & $4(40 \%)$ \\
\hline \multirow[t]{2}{*}{ Calamia et al., 2011} & \multirow[t]{2}{*}{ IRT based } & Original & $12(60 \%)$ & $8(40 \%)$ & $5(50 \%)$ & $5(50 \%)$ \\
\hline & & Mirror reversed & $14(70 \%)$ & $6(30 \%)$ & $7(70 \%)$ & $3(30 \%)$ \\
\hline
\end{tabular}

patients), this variable was entered in the analysis of variance as a covariate. ${ }^{1}$

The main effect of the JLO form was significant, $F(8,216)=$ 7.9, $p<.001, \eta_{p}^{2}=0.23$. The post hoc test (Newmann-Keuls method) revealed that the forms with items 1-10 and 1-20 resulted in significantly higher mean percentages of correctly answered items $(77 \%$ and $72 \%)$ than all the other forms, all $p$ s $<.001$. The performance with the form including the first 10 items was significantly better than that with the form including the first 20 items, whereas the form with items 11-30 was that producing the lowest mean percentage score $(57 \%)$, all $p \mathrm{~s}<.001$. This is consistent with the idea that JLO items increase in difficulty as the test progresses (cf., Calamia et al., 2011) and that the most difficult items are in the last ordinal positions.

The main effect of item version was also significant, $F(1,27)=$ 11.11, $p<.005, \eta_{p}^{2}=0.29$ : On the whole, performance was better with mirror-reversed than with original items (68\% vs. 63\%). However, item version significantly interacted with group, $F(1$, $27)=10.145, p<.005, \eta_{p}^{2}=0.28$ : The mean percentages of original and mirror-reversed items correctly identified by LHD versus RHD patients were $66 \%$ versus $60 \%$ and $66 \%$ versus $69 \%$, respectively. The three-way interaction was not significant, $F(8$, 216) $=0.13, p=1.0, \eta_{p}^{2}=0.005$. Indeed, the same trend was observed for all forms. As shown in Figure 2, in all the short forms, just as in the full form, a relative advantage of LHD patients over RHD patients was observed for the original items. This difference between the two groups of patients decreased or turned into a relative advantage of RHD patients over LHD patients for the mirror-reversed items.

Planned (interactive) contrast revealed that for two short forms, Items $1-10$ and Form $\mathrm{Q}$, the critical interaction between item version and group felt short of significance, $F=4.2, p=.0502$, and $F=3.95, p=.0571$, respectively. However, for the other six short forms, the Version $\times$ Group interaction was significant, all $F_{\mathrm{s}} \geq 4.78, p \mathrm{~s} \leq .038$.

The raw scores of six short forms (Odd Form, Even Form, Items 11-30, Form Q, Form S and the IRT-based form) could be con- verted in full form scores that, once corrected for age and gender, allowed us to classify patients' performances as either unimpaired or impaired. ${ }^{2}$ A cutoff score of $>20$ (as unimpaired) was used (Benton et al., 1983; see also Spencer et al., 2013; Gullett et al., 2013). Table 3 shows the number and percentages of LHD and RHD patients with impaired/unimpaired scores in the two item versions of the JLO full form and these six short forms. On the whole, among RHD patients the percentage of impaired performance tended to be higher with original items than with mirrorreversed items. The difference between the two items versions was smaller among the LHD patients.

Finally, a by-item analysis was performed to evaluate whether the different pattern of LHD and RHD performances with the original and mirror-reversed JLO versions could be attributed to specific items. Figure 3 depicted, for each item, the difference between the mean scores of the original and mirror-reversed versions obtained by LHD and RHD patients. Values opposite in sign are in correspondence of items for which LHD and RHD patients performed differently, depending on the item version. The difference between these values is also depicted. The largest differences are observed for items V20, V23, V25, and V29. For all these four items, performances of LHD patients were better with the original version, whereas performances of RHD patients were better with the mirror-reversed version. Planned (interactive) contrasts revealed that, for these items only, the inversion of the relative advantage of LHD over RHD patients from the original to the

\footnotetext{
${ }^{1}$ It is worth noticing that, despite the higher education level of RHD patients (which, ceteris paribus, should have favored these patients; cf., Benton et al., 1983), in all short forms, just as in the full form, a relative advantage of LHD patients over RHD patients was observed for the original items. The difference between the performances of the two groups of patients either reversed or disappeared in the mirror-reversed version of the test, thus suggesting that it cannot be attributed to differences in the education level between LHD and RHD patients.

${ }^{2}$ For the forms with items $1-10$ and 1-20, neither procedures to convert short form scores to full form scores nor appropriate cutoff scores are available.
} 

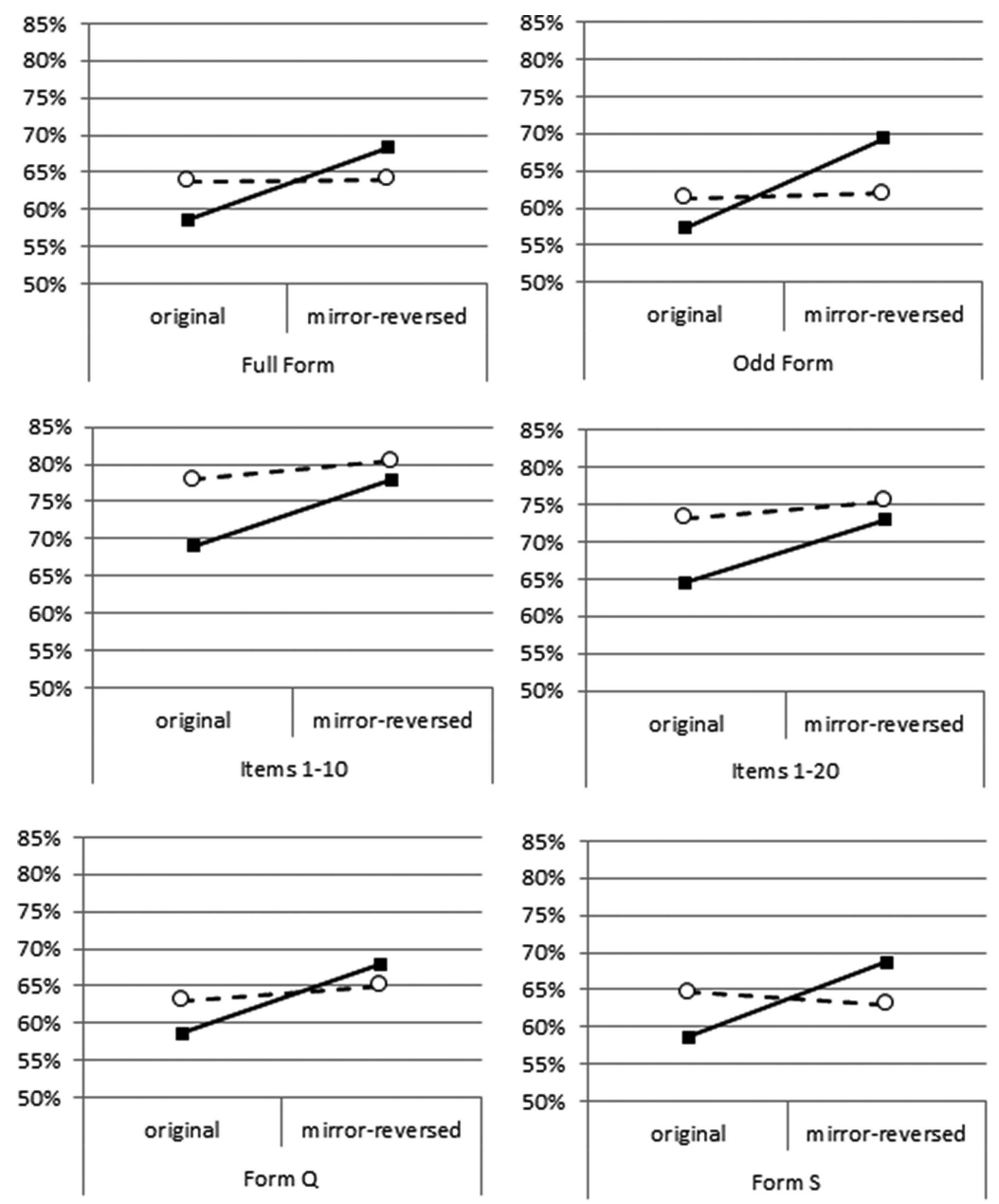
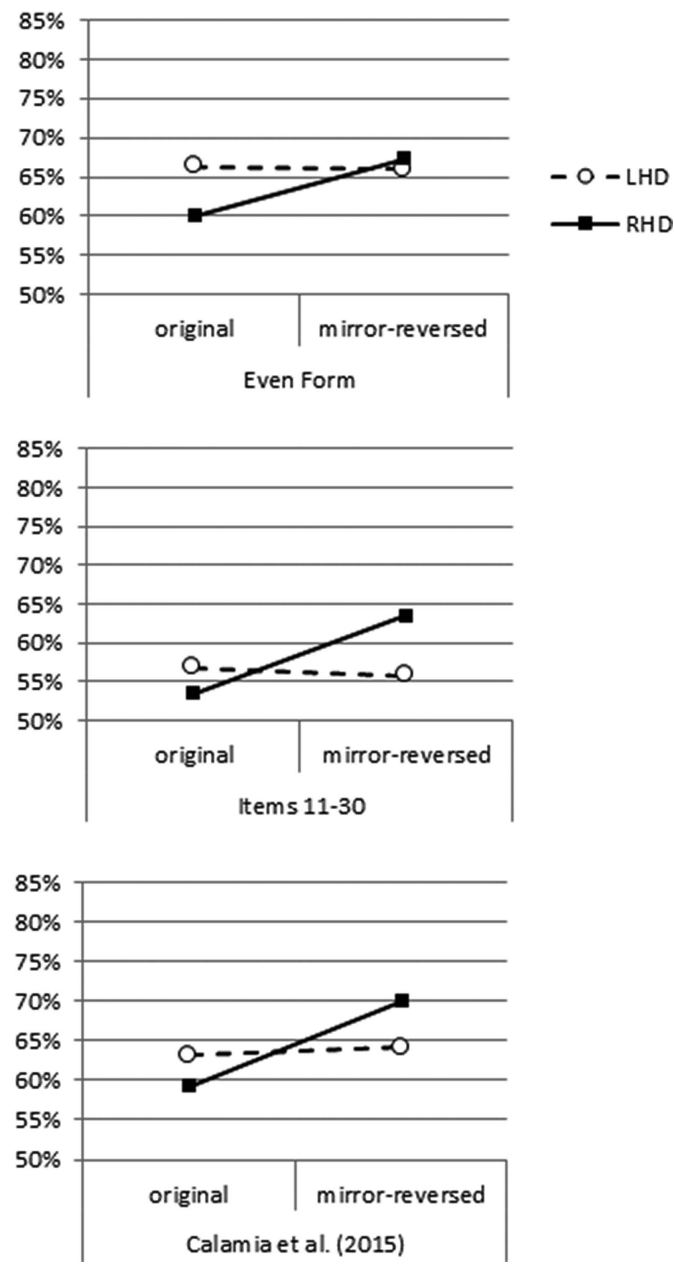

Figure 2. Percentages of original and mirror-reversed items of the full and short forms of the Judgment of Line Orientation test that were correctly answered by left hemisphere-damaged patients (LHD) and right hemispheredamaged patients (RHD).

mirror-reversed version is significant (all $F_{\mathrm{s}} \geq 6.22, p \mathrm{~s} \leq .019$, $\eta_{p}^{2} \geq 0.18$ ). Notably, the two short forms for which the Version $\times$ Group interaction fell short of significance (Items 1-10 and Form Q) contain either none or just one of these critical items.

For all the other items, the patterns of performances of LHD and RHD patients with the two item versions did not significantly differ (all $F \mathrm{~s} \leq 2.7$; all $p \mathrm{~s} \geq .11$ ).

It appears clear from Figure 3 that the Version $\times$ Group effect is higher for items in the last ordinal positions. As mentioned above, items toward the end of the test should be the most difficult ones. Indeed, the four items showing the largest differences between the LHD and RHD patients' performance in the two item versions are the 17th, 24th, 27th, and 28th most difficult items according to the difficulty estimates by Calamia et al. (2011). Based on the accuracy scores of our sample of brain-damaged patients, they were the 19th, 23rd, 28th, and 30th most difficult items.

A closer look reveals that the original version of all four items contains line 3 (i.e., a left line forming an angle of $36^{\circ}$ with the horizontal line; see Figure 1), which, being oblique and far from either the horizontal and vertical, is quite difficult to identify (Appelle, 1972; Treccani et al., 2005). This line proved to be particularly difficult for RHD patients: The mean accuracy scores for line 3 obtained in these four items by LHD and RHD patients were 0.6 and 0.4 , respectively. In contrast, in the mirror-reversed version of these items, the right $36^{\circ}$ line (line 9) resulting from mirror reversing line 3, was particularly difficult to judge for LHD patients: The mean scores for this line obtained by LHD and RHD patients were 0.4 and 0.7 , respectively.

\section{Discussion}

In the present study, we analyzed the eight short forms of JLO proposed in the literature (Spencer et al., 2013). Our aim was to establish whether these short forms are spatially asymmetric, just like the full form (Treccani et al., 2005), and to examine the impact of the possible asymmetries on the performances of unilateral brain-damaged patients. To this end, we reanalyzed the data of Treccani et al. (2005) and compared performances of LHD and RHD patients on the short forms and their mirror-reversed ver- 


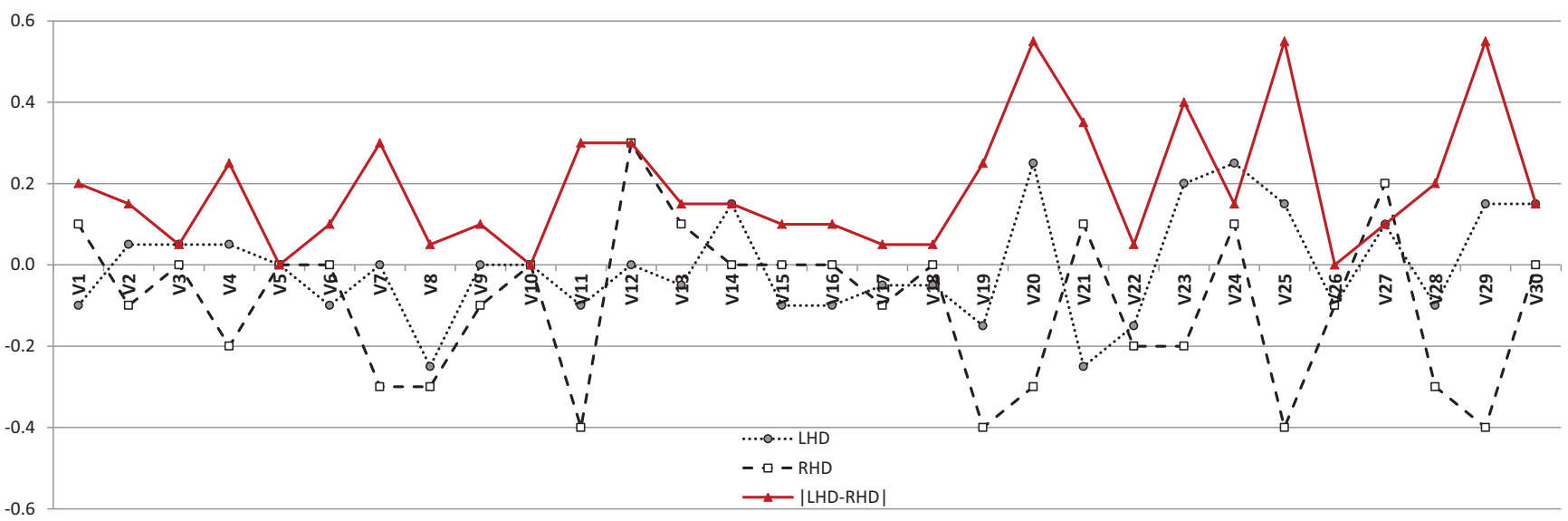

Figure 3. Dashed lines represent the differences between mean scores of original and mirror-reversed items (o-mr $\Delta$ s) of the Judgment of Line Orientation test for left hemisphere-damaged (LHD) and right hemispheredamaged (RHD) patients. Positive and negative values of these o-mr $\Delta$ s indicate higher scores for original and mirror-reversed items, respectively. The red solid line represents the difference, in absolute value, between o-mr $\Delta \mathrm{s}$ of LHD and RHD patients. The highest values are in correspondence to items for which the difference between LHD and RHD patients is opposite in the original and mirror-reversed versions. See the online article for the color version of this figure.

sions. This is the best way to uncover possible left-right asymmetries underlying differences between LHD and RHD patients' performances because, in the presence of such asymmetries, different patterns of performance of LHD and RHD patients would be observed, depending on the item version (i.e., original or mirror reversed).

Results from the present study clearly show that the JLO short forms are largely asymmetric, exactly like, or even more than, the full form. Not only are left- and right-sided lines of different types but also the number of lines itself differs between the two spatial sides. These structural asymmetries seem to interact with the attentional biases due to unilateral brain lesions. In six of the eight analyzed short forms, a relative advantage of LHD patients over RHD patients was found for the original items, which significantly decreased or turned into a relative advantage of RHD patients over LHD patients for the mirror-reversed items. As clearly appears from Figure 2, this inversion is mainly due to an increase of RHD patients' performance with the mirror-reversed items. The two short forms for which such an inversion does not reach significance (Items 1-10 and Form Q) contain either none or just one of the four critical items (V20, V22, V26, and V29). All these items include a $36^{\circ}$ line. Unilateral brain-lesioned patients in our sample faced difficulties with this line orientation when presented in their contralesional side: RHD patients with line 3 in the original version of these items and LHD patients with line 9 in the mirrorreversed version.

Based on these results, one may argue that removing these four items could be a sensible solution to the problem of JLO asymmetries distorting the assessment of brain-damaged patients' visuospatial abilities. In this way, however, we would remove four of the most difficult, and potentially discriminating, items. In addition, it is worth noticing that, even for the JLO short version that does not contain any of these items, the pattern of patients' performance in the two item versions is similar to that observed with the other forms.
Indeed, even without such items, both the full and short JLO forms examined here would remain asymmetric (i.e., they would continue to show a different distribution of left and right lines). Without these four items, in our sample the difference between LHD and RHD patients' performances on the two item versions is smaller, but we cannot predict how the still-present structural asymmetries will interact with the possible attentional biases of other patients. To really control for the effect of such asymmetries, they should be fully removed. This obviously implies an a priori selection of the to-be-presented items: Items should be properly selected to ensure that the final form is balanced.

The IRT-based short form proposed by Calamia et al. (2011) is no doubt interesting and has been reported to have good reliability when used with patients without neurological diseases (Spencer et al., 2013). However, the results of the present study clearly show that this form is not suitable to test patients with possible unilateral attentional biases due to unilateral brain damage. We cannot know in advance which items will be presented and whether the item selection algorithm will result in a left-right balanced test form. The selected items depend on the responses of the examinee and results are completely unpredictable.

We acknowledge that, in both clinical and research settings, a fast and easy-to-administer instrument for the assessment of visuospatial perception is needed. JLO can still be used to assess the ability to judge angular relationships. However, whatever JLO form is used, any asymmetries between left- and right-sided lines in the stimulus arrays must be avoided. The presence of left-right asymmetries in the current set of JLO items is undisputable, and their detrimental impact on unilateral brain-damaged patients' performance is clear (Treccani et al., 2005, 2011); therefore, they cannot be neglected anymore in the development of any possible form of JLO. To obtain a short, but balanced, version of JLO, in which the spatial features of the lines in the left and right halves of the items are comparable, we propose to select five to 10 items among those of the original set, taking care to include items of all the possible 
difficulty levels, and present them together with their mirror images.

\section{References}

Appelle, S. (1972). Perception and discrimination as a function of stimulus orientation: The "oblique effect" in man and animals. Psychological Bulletin, 78, 266-278. http://dx.doi.org/10.1037/h0033117

Basso, A., Capitani, E., \& Laiacona, M. (1987). Raven's coloured progressive matrices: Normative values on 305 adult normal controls. Functional Neurology, 2, 189-194.

Benton, A. L., Hamsher, K., Varney, N. R., \& Spreen, O. (1983). Contributions to neuropsychological assessment. New York, NY: Oxford University Press.

Benton, A. L., Varney, N. R., \& Hamsher, K. D. (1978). Visuospatial judgment. A clinical test. Archives of Neurology, 35, 364-367. http:// dx.doi.org/10.1001/archneur.1978.00500300038006

Calamia, M., Markon, K., Denburg, N. L., \& Tranel, D. (2011). Developing a short form of Benton's Judgment of Line Orientation Test: An item response theory approach. Clinical Neuropsychologist, 25, 670-684. http://dx.doi.org/10.1080/13854046.2011.564209

Colombo, A., De Renzi, E., \& Faglioni, P. (1976). The occurrence of visual neglect in patients with unilateral cerebral disease. Cortex, 12, 221-231. http://dx.doi.org/10.1016/S0010-9452(76)80003-2

Cubelli, R. (2017). Definition: Spatial neglect. Cortex, 92, 320-321. http:// dx.doi.org/10.1016/j.cortex.2017.03.021

Gullett, J. M., Price, C. C., Nguyen, P., Okun, M. S., Bauer, R. M., \& Bowers, D. (2013). Reliability of three Benton Judgment of Line Orientation short forms in idiopathic Parkinson's disease. Clinical Neuropsychologist, 27, 1167-1178. http://dx.doi.org/10.1080/13854046.2013.827744

Hamsher, K., Capruso, D. X., \& Benton, A. (1992). Visuospatial judgment and right hemisphere disease. Cortex, 28, 493-495. http://dx.doi.org/10 1016/S0010-9452(13)80157-8

Lezak, M. D., Howieson, D. B., Bigler, E. D., \& Tranel, D. (2012). Neuropsychological assessment (5th ed.). New York, NY: Oxford University Press.

Moore, T. M., Scott, J. C., Reise, S. P., Port, A. M., Jackson, C. T., Ruparel, K., . . Gur, R. C. (2015). Development of an abbreviated form of the Penn Line Orientation Test using large samples and computerized adaptive test simulation. Psychological Assessment, 27, 955-964. http:// dx.doi.org/10.1037/pas0000102

Mount, D. L., Hogg, J., \& Johnstone, B. (2002). Applicability of the 15 -item versions of the Judgement of Line Orientation Test for individuals with traumatic brain injury. Brain Injury, 16, 1051-1055. http://dx .doi.org/10.1080/02699050210154259
Qualls, C. E., Bliwise, N. G., \& Stringer, A. Y. (2000). Short forms of the Benton Judgment of Line Orientation Test: Development and psychometric properties. Archives of Clinical Neuropsychology, 15, 159-163.

Raven, J. C. (1965). Guide to using the coloured progressive matrices. London, United Kingdom: H. K. Lewis.

Spencer, R. J., Wendell, C. R., Giggey, P. P., Seliger, S. L., Katzel, L. I., \& Waldstein, S. R. (2013). Judgment of Line Orientation: An examination of eight short forms. Journal of Clinical and Experimental Neuropsychology, 35, 160-166. http://dx.doi.org/10.1080/13803395.2012.760535

Spinnler, H., \& Tognoni, G. (1987). Standardizzazione e taratura italiana di test neuropsicologici. Italian Journal of Neurological Sciences, 8(Suppl.), 1-120.

Tranel, D., Vianna, E., Manzel, K., Damasio, H., \& Grabowski, T. (2009). Neuroanatomical correlates of the Benton Facial Recognition Test and Judgment of Line Orientation Test. Journal of Clinical and Experimental Neuropsychology, 31, 219-233. http://dx.doi.org/10.1080/1380339 0802317542

Treccani, B., \& Cubelli, R. (2011). The need for a revised version of the Benton judgment of line orientation test. Journal of Clinical and Experimental Neuropsychology, 33, 249-256. http://dx.doi.org/10.1080/1380 3395.2010 .511150

Treccani, B., Torri, T., \& Cubelli, R. (2005). Is judgement of line orientation selectively impaired in right brain damaged patients? Neuropsychologia, 43, 598-608. http://dx.doi.org/10.1016/j.neuropsychologia 2004.07.008

van der Berg, E., \& Ruis, C. (2017). Space in neuropsychological assessment. In A. Postma \& I. J. M. van der Ham (Eds.), Neuropsychology of space: Spatial functions of the human brain (pp. 361-378). Cambridge, MA: Elsevier Academic Press. http://dx.doi.org/10.1016/B978-0-12-8016381.00010-0

Venderploeg, R. D., LaLone, L. V., Greblo, P., \& Schinka, J. A. (1997). Odd-even short forms of the judgment of line orientation test. Applied Neuropsychology, 4, 244-246. http://dx.doi.org/10.1207/s15324826an 0404_6

Winegarden, B. J., Yates, B. L., Moses, J. A., Benton, A. L., \& Faustman, W. O. (1998). Development of an optimally reliable short form for Judgment of Line Orientation. Clinical Neuropsychologist, 12, 311-314 http://dx.doi.org/10.1076/clin.12.3.311.1992

Woodard, J. L., Benedict, R. H. B., Salthouse, T. A., Toth, J. P., Zgaljardic, D. J., \& Hancock, H. E. (1998). Normative data for equivalent, parallel forms of the Judgment of Line Orientation Test. Journal of Clinical and Experimental Neuropsychology, 20, 457-462. http://dx.doi.org/10.1076/ jcen.20.4.457.1470

Received April 5, 2018

Revision received August 19, 2018

Accepted October 28, 2018 